\title{
Treatment Pattern and Economic Burden of Refractory Gastroesophageal Reflux Disease Patients in Korea
}

\author{
Susan Park, ${ }^{1}$ Jin-Won Kwon, ${ }^{1}$ Joong-Min Park, ${ }^{2}$ Sungsoo Park, ${ }^{3}$ and Kyung Won Seo ${ }^{4 *}$ \\ ${ }^{1}$ College of Pharmacy and Research Institute of Pharmaceutical Sciences, Kyungpook National University, Daegu, Korea; ${ }^{2}$ Department of \\ Surgery, Chung-Ang University College of Medicine, Seoul, Korea; ${ }^{3}$ Department of Surgery, Korea University College of Medicine, Seoul, Korea; \\ and ${ }^{4}$ Department of Surgery, Kosin University College of Medicine, Busan, Korea
}

\section{Background/Aims}

The prevalence of gastroesophageal reflux disease (GERD) has increased in Korea, and the economic burden of this chronic disease is substantial. We aim to investigate the treatment pattern and economic burden in Korea of refractory GERD requiring long-term use of proton pump inhibitors (PPIs).

\section{Methods}

We conducted a cross-sectional analysis of nationally representative sample data obtained from 2012 to 2016 and provided by the Health Insurance Review and Assessment Service. The participants were 86936 in the medication group and 40 in the surgery group. Age- and sex-standardized prevalence of refractory GERD requiring PPIs medication for more than 12 weeks were analyzed. Generalized linear models were used to estimate cost ratios for comparing the medical costs of the surgery and medication groups after adjustment for demographics and comorbidity.

\section{Results}

The prevalence of refractory GERD increased from $1.2 \%$ in 2012 to $1.9 \%$ in 2016, and the estimated total number of GERD patients increased from 402000 to 736000 during this time period. The medical expenditures of the surgery group within 90 days of antireflux surgery (ARS) were 16.9-fold higher compared to those of the medication group; the significant difference in medical costs between the 2 groups disappeared after 90 days post-surgery.

\section{Conclusions}

The prevalence of refractory GERD requiring long-term use of PPIs has been trending upwards recently. Nevertheless, ARS is very rarely performed. Considering the increasing medical costs of long-term PPI use, further cost-effectiveness analysis is needed to compare ARS and PPI therapy for the treatment of GERD in Korea.

(J Neurogastroenterol Motil 2020;26:281-288)

Key Words

Fundoplication; Gastroesophageal reflux; Proton pump inhibitors

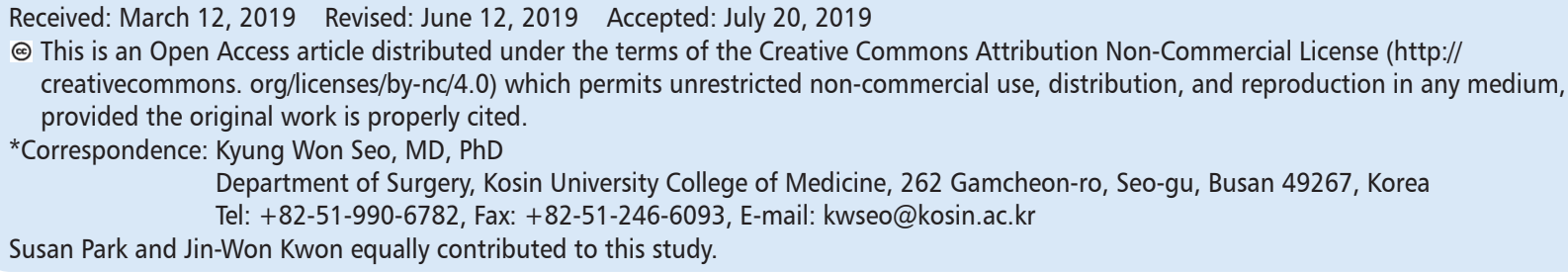




\section{Introduction}

Gastroesophageal reflux disease (GERD), a common disease in which the stomach contents reflux into the esophagus, can cause esophagitis and daily life disorders. In North American studies, the GERD prevalence has been estimated as 18.1-27.8\%, in European studies, 8.8-25.9\%, and in Asian studies, 2.5-7.8\%. ${ }^{1}$ Although the prevalence of GERD has been lower in Asian than in Western populations, it has recently shown a rapidly increasing trend in Asia. ${ }^{2}$ In Korea, the prevalence of GERD has increased from $4.6 \%$ to $7.3 \%$ between 2005 and 2008.,

Because of the high prevalence and chronic nature of the disease, the impact of GERD on health care utilization and medical expenditure have been considerable. ${ }^{5}$ In the United States (US), GERD is the most common gastrointestinal (GI) disease diagnosed in outpatient clinic visits, ${ }^{6}$ and the medical costs were esti-

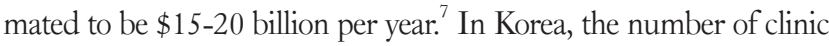
patients treated for GERD more than tripled from 2000 to 2011 ( $\mathrm{n}=990000$ in 2000 and $\mathrm{n}=3110000$ in 2011). The medical expenditure for GERD was approximately $\$ 1.5$ billion out of the $\$ 36$ billion expended for all GI disease in 2007 . $^{8}$ Considering the trend of increasing prevalence, the expenditures for GERD can be expected to rise much higher.

Treatment for GERD includes lifestyle modification, medications such as antacids or proton pump inhibitors (PPIs), and antireflux surgery (ARS). Most patients with GERD receive PPIs to manage the symptoms of the disease. ${ }^{9}$ Unfortunately, GERD symptoms reportedly persist in $30-60 \%$ of patients who receive PPIs. ${ }^{8,10}$ Moreover, considerable empirical evidence has accumulated about various adverse events associated with long-term use of PPIs, such as osteoporosis, ischemic heart disease, chronic kidney injury, and dementia. ${ }^{11}$ Despite the widespread use of PPIs, prevalence data on the long-term use of PPIs in Korea have been lacking.

ARS is an alternative for the treatment of GERD in cases of severe disease requiring long-term use of PPIs and recurrent or intractable disease. ${ }^{12}$ The clinical guidelines for surgical and medical treatments for GERD are well-established, ${ }^{12}$ and the utilization of ARS is substantial in Western countries. ${ }^{13-15}$ However, ARS has been rarely performed in Korea.

In order to improve treatment effectiveness and reduce unnecessary medical expenditures, it is important to explore the treatment patterns of refractory GERD in Korea and estimate their medical costs. Thus, we aim to use nationally representative data to investigate: (1) numbers of patients undergoing ARS and receiving long- term treatment with PPIs, (2) PPI prescription patterns among long-term PPI users, and (3) medical expenditures according to the treatment type (ARS and PPIs).

\section{Materials and Methods}

\section{Study Subjects}

We conducted a cross-sectional analysis using nationally representative sample data provided by the Health Insurance Review and Assessment Service (HIRA). The HIRA claims data, collected for reimbursement of national healthcare insurance and medical aid, include patients' diagnoses, treatment, procedures, surgical history, and prescription drugs. HIRA provides several datasets using different sampling strategies to ensure dataset reliability and representativeness of the specific target population. The HIRANational Patient Sample (NPS), a random selection of $3.0 \%$ of all patients, represents the total population that visits medical institutions each year. The HIRA-National Inpatient Sample (NIS), a random selection of $13.0 \%$ of total inpatients annually, is suitable for analysis of inpatients. ${ }^{8,9}$ We used the HIRA-NPS for the analysis of medication therapy for GERD, and we used the HIRA-NIS for the analysis of GERD surgery. The NPS from 2012 to 2016 included 11 cases of surgical treatment for GERD; the NIS from this period included 40 such cases.

Figure 1 presents the study population selection procedure. For this study, we selected 86936 participants for the medication group and 40 participants for the surgery group. The NPS and NIS were released after anonymization and de-identification of the data; therefore, informed consent could not be obtained from participants individually. This study was approved by the Institutional Review Board of Kosin University Gospel Hospital (IRB No. KUGH 2018-02-013).

\section{Variables}

The surgical group was composed of patients diagnosed with GERD who underwent fundoplication. The diagnosis of GERD was defined according to the "International Classification of Disease, 10th edition, clinical modification (ICD-10-CM)" as follows: K20.0, K20.9, K21.0, and K21.9. Fundoplication was confirmed using the corresponding surgical codes.

The medication group included patients with refractory GERD who required medication with PPIs for more than 12 weeks to treat GERD symptoms. A consecutive prescription was defined as having the next prescription of PPIs within 7 days of the 
A

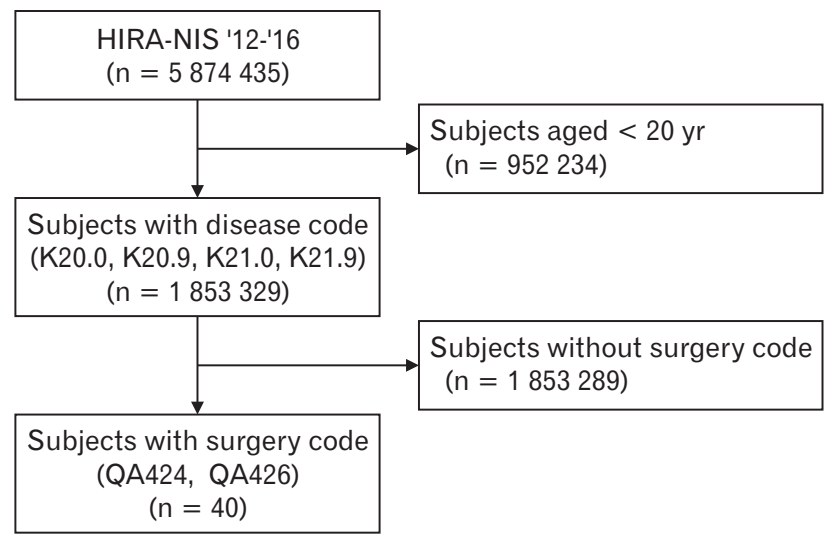

B

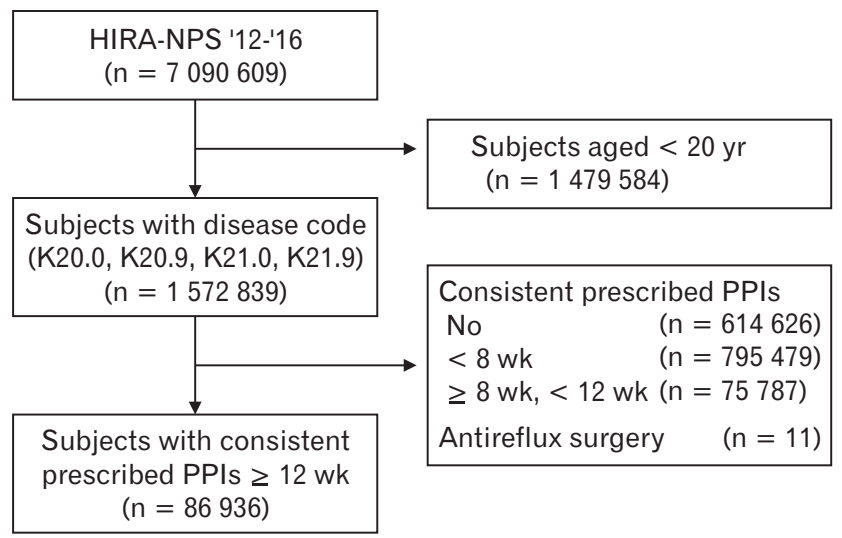

Figure 1. Flow chart for the study population selection procedure. (A) Medication group. (B) Surgery group. HIRA-NIS, the Health Insurance Review and Assessment Service-National Inpatient Sample; HIRA-NPS, the Health Insurance Review and Assessment Service-National Patient Sample; PPI, proton pump inhibitor.

last date of the prescription period. We calculated the average prescribed daily dose (PDD) of PPIs during the primary consecutive prescription period of 12 weeks and then categorized these doses into 3 groups: $<$ standard dose, standard to $<$ double dose, and $\geq$ double dose.

Medical expenditure was defined as the total costs of GERD treatment in the medical care institute covered by the insurer and beneficiary. Average monthly costs for each group were calculated as total medical costs divided by the number of follow-up months to account for the difference in observation time for GERD treatments. The follow-up period for the surgery group was calculated from the day of operation to December 31 in each year. In the medication group, the follow-up time was counted from the end date of the primary consecutive prescription of 12 weeks to December 31 . Medical costs were presented as US dollars, which were calculated based on an exchange rate of Korean Republic won $1000=\$ 1$.

Health status at baseline was measured using the Charlson $\mathrm{Co}_{\mathrm{O}}$ morbidity Index (CCI), ${ }^{10}$ which is scored using 17 types of chronic diseases: myocardial infarction, congestive heart failure, peripheral vascular disease, cerebrovascular disease, dementia, chronic pulmonary disease, rheumatic disease, peptic ulcer disease, mild liver disease, moderate or severe liver disease, non-complicated diabetes, complicated diabetes, hemiplegia or paraplegia, renal disease, any malignancy except skin cancer, metastatic solid tumor, and acquired immunodeficiency syndrome. These chronic diseases were defined based on the ICD-10 codes in the medical treatment data and then each disease was weighted based on its severity.

\section{Analysis}

We analyzed the prevalence and $95 \%$ confidence intervals (CIs) of patients who were prescribed PPIs for more than 12 weeks from 2012 to 2016. The overall prevalence was calculated based on the population over age 20 years in Korea and standardized by age and sex using the distribution of the 2015 census population in Korea. The prevalence was also analyzed by sex and age subgroups. The Cochran-Armitage trend test was conducted to confirm the increasing prevalence. To estimate the number of patients in Korea, we calculated the weighted number of patients prescribed PPIs more than 12 weeks using the sampling weight in the HIRA-NPS.

The mean and standard deviation of the medical costs were estimated for inpatients, outpatients, and prescribed drug costs (PPI costs and other drug costs). We estimated the mean total medical costs in the surgery group and this was split into 2 parts according to the follow-up time of 3 months post-operation to distinguish the cost of surgery from that of GERD treatment. For the medication group, we calculated the mean costs according to the PDD of PPIs.

A generalized linear model (GLM) was used to compare the medical expenditures of the medication and surgery groups after adjusting for confounding variables such as demographic information and comorbidities. Log link and gamma distributions were selected in the GLM to reflect the right-skewed distribution of medical expenditure. ${ }^{11}$ We considered the follow-up month as an offset variable because participants had different follow-up periods. The cost ratios and 95\% CIs using the GLM are calculated. All statistical analyses were performed using SAS version 9.4 (SAS Institute Inc, Cary, NC, USA). 


\section{Results}

Table 1 shows the distributions of the demographics and comorbidities for the medication and surgery groups. The number of GERD patients who received PPIs for at least 12 weeks was 86 936, which corresponded to $9.1 \%$ of the GERD patients receiving

Table 1. General Distribution of Study Participants: The Health Insurance Review and Assessment Service-National Patient Sample and the Health Insurance Review and Assessment Service-National Inpatient Sample (2012-2016)

\begin{tabular}{|c|c|c|c|c|}
\hline \multirow{3}{*}{ Variables } & \multicolumn{2}{|c|}{$\begin{array}{l}\text { HIRA-NPS } \\
(2012-2016)\end{array}$} & \multicolumn{2}{|c|}{$\begin{array}{l}\text { HIRA-NIS } \\
(2012-2016)\end{array}$} \\
\hline & \multicolumn{2}{|c|}{$\begin{array}{c}\text { Medication group } \\
\text { (prescribed PPIs } \geq 12 \text { weeks) }\end{array}$} & \multicolumn{2}{|c|}{$\begin{array}{l}\text { Surgery group } \\
\text { (fundoplication) }\end{array}$} \\
\hline & $\mathrm{n}$ & $\%(\mathrm{SE})$ & $\mathrm{n}$ & $\%(\mathrm{SE})$ \\
\hline Total & 86936 & $100.00(0.00)$ & 40 & $100.00(0.00)$ \\
\hline \multicolumn{5}{|l|}{ Sex } \\
\hline Men & 39388 & $45.31(0.17)$ & 20 & $50.00(8.01)$ \\
\hline Women & 47548 & $54.69(0.17)$ & 20 & $50.00(8.01)$ \\
\hline \multicolumn{5}{|l|}{ Age (yr) } \\
\hline $20-39$ & 3046 & $3.50(0.06)$ & 16 & $40.00(7.84)$ \\
\hline $40-59$ & 26967 & $31.02(0.16)$ & 14 & $35.00(7.64)$ \\
\hline$\geq 60$ & 56923 & $65.48(0.16)$ & 10 & $25.00(6.93)$ \\
\hline \multicolumn{5}{|l|}{ CCI score } \\
\hline $0-1$ & 21011 & $24.17(0.15)$ & 12 & $30.00(7.34)$ \\
\hline $2-3$ & 30931 & $35.58(0.16)$ & 23 & $57.50(7.92)$ \\
\hline$\geq 4$ & 34994 & $40.25(0.17)$ & 5 & $12.50(5.30)$ \\
\hline
\end{tabular}

HIRA-NIS, the Health Insurance Review and Assessment Service-National Inpatient Sample; HIRA-NPS, the Health Insurance Review and Assessment Service-National Patient Sample; PPI, proton pump inhibitors; CCI, Charlson Comorbidity Index.
PPIs ( $\mathrm{n}=958213), 5.5 \%$ of the total number of GERD patients ( $\mathrm{n}$ $=1572839$ ), and $1.6 \%$ of the total population aged 20 and over $(\mathrm{n}=5611025)$. The numbers of male and female subjects were similar in both groups, but there were more subjects with older age and a high CCI score in the medication group than in the surgery group.

Figure 2 presents the age- and sex-standardized prevalence in the adult population and estimated numbers of patients prescribed PPIs for more than 12 weeks in the 5-year study period. The prevalence increased from 2012 to 2016 (11.8 in 2012, 19.1 in 2016, per 1000 people), which was statistically significant in the CochranArmitage trend test $(P$ for trend $<0.001)$. As the prevalence increased, the numbers of patients also rose from 402000 in 2012 to 736000 in 2016.

Figure 3 shows the prevalence of patients prescribed PPIs more than 12 weeks by age and sex. The prevalence was higher in women than in men, which tended to increase similarly. The prevalence was notably higher in adults aged over 60 years than in those aged 20-50 years. The results of the Cochran-Armitage trend test showed that the prevalence of all sexes and ages showed a statistically significant increase in the 5-year study period.

Table 2 presents the proportions of PDD levels during the primary 12-week consecutive prescription period of PPIs in the medication group. The percentages of patients prescribed PPIs below the standard dose, between the standard and double doses, and at or above the double dose were $85.6-89.1 \%, 10.4-13.5 \%$, and $0.5-0.9 \%$, respectively. Hence, the proportion of patients prescribed PPIs below the standard dose decreased from 2012 to 2016, whereas the proportion of patients prescribed PPIs at or above the double dose increased almost 2-fold.

Table 3 shows the average costs for inpatients, outpatients, PPIs, and other GERD drugs according to therapy type. The medical costs of the surgery group are presented according to the 2

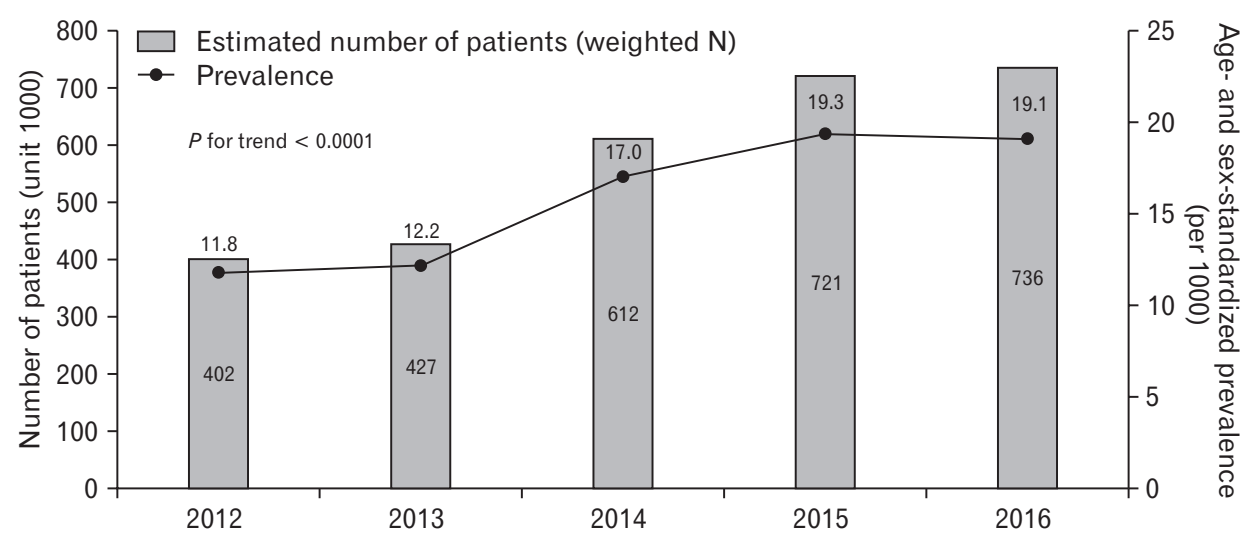

Figure 2. Age- and sex-standardized prevalence and estimated number of patients prescribed proton pump inhibitors (PPIs) for more than 12 weeks. 
A

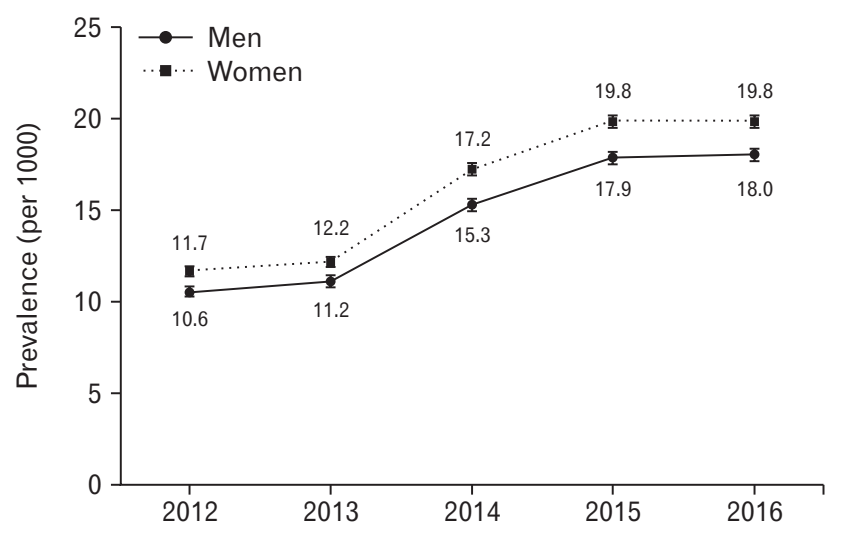

B

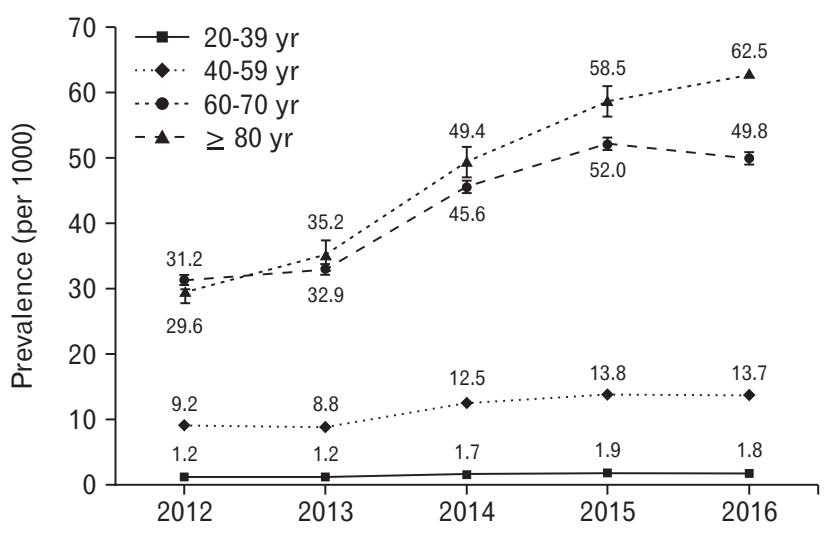

Figure 3. Age- and sex-specific prevalence of patients prescribed proton pump inhibitors (PPIs) for more than 12 weeks. (A) Sex-specific prevalence. (B) Age-specific prevalence.

Table 2. Distributions of the Prescribed Daily Dose of Proton Pump Inhibitors in Patients Prescribed Proton Pump Inhibitors for More Than 12 Weeks

\begin{tabular}{|c|c|c|c|c|c|c|c|c|c|c|}
\hline \multirow{2}{*}{ Variables } & \multicolumn{2}{|r|}{2012} & \multicolumn{2}{|r|}{2013} & \multicolumn{2}{|r|}{2014} & \multicolumn{2}{|r|}{2015} & \multicolumn{2}{|r|}{2016} \\
\hline & $\mathrm{n}$ & $\%(\mathrm{SE})$ & $\mathrm{n}$ & $\%(\mathrm{SE})$ & $\mathrm{n}$ & $\%(\mathrm{SE})$ & $\mathrm{n}$ & $\%(\mathrm{SE})$ & $\mathrm{n}$ & $\%(\mathrm{SE})$ \\
\hline PDD of PPIs & 12059 & $100.00(0.00)$ & 12802 & $100.00(0.00)$ & 18360 & $100.00(0.00)$ & 21643 & $100.00(0.00)$ & 22072 & $100.00(0.00)$ \\
\hline$<$ standard dose & 10747 & $89.12(0.28)$ & 11493 & $89.78(0.27)$ & 16178 & $88.12(0.24)$ & 18871 & $87.19(0.23)$ & 18893 & $85.60(0.24)$ \\
\hline Standard to $<$ double dose & 1249 & $10.36(0.28)$ & 1249 & $9.76(0.26)$ & 2089 & $11.38(0.23)$ & 2630 & $12.15(0.22)$ & 2978 & $13.49(0.23)$ \\
\hline$\geq$ double dose & 63 & $0.52(0.07)$ & 60 & $0.47(0.06)$ & 93 & $0.51(0.05)$ & 142 & $0.66(0.05)$ & 201 & $0.91(0.06)$ \\
\hline
\end{tabular}

PDD, prescribed daily dose; PPI, proton pump inhibitor.

Table 3. Medical Costs of Gastroesophageal Reflux Disease Treatment in the Medication and Surgery Groups (Unit: Dollars)

\begin{tabular}{|c|c|c|c|c|c|c|c|}
\hline Variables & $\mathrm{n}$ & $\begin{array}{l}\text { A. Inpatient } \\
\text { costs }\end{array}$ & $\begin{array}{l}\text { B. Outpatient } \\
\text { costs }\end{array}$ & $\begin{array}{l}\text { C. PPI } \\
\text { costs }\end{array}$ & $\begin{array}{l}\text { D. Other drug } \\
\text { costs }\end{array}$ & $\begin{array}{l}\text { E. Follow-up } \\
\text { months }\end{array}$ & $\begin{array}{l}\text { Total monthly costs } \\
(\mathrm{A}+\mathrm{B}+\mathrm{C}+\mathrm{D}) / \mathrm{E}\end{array}$ \\
\hline \multicolumn{8}{|l|}{ Surgery group } \\
\hline Total & 40 & $4591.7(403.90)$ & $232.4(42.83)$ & $37.7(12.78)$ & $200.0(111.51)$ & $6.7(0.61)$ & $1423.9(259.22)$ \\
\hline \multicolumn{8}{|l|}{$\begin{array}{l}\text { Number of days from } \\
\text { surgery }\end{array}$} \\
\hline$\leq 90$ & 40 & $4509.7(390.74)$ & $99.1(19.57)$ & $13.1(4.26)$ & $153.5(110.67)$ & $2.6(0.11)$ & $2071.6(240.17)$ \\
\hline$>90^{\mathrm{a}}$ & 30 & $109.4(109.42)$ & $177.7(48.37)$ & $32.8(14.22)$ & $62.1(23.41)$ & $5.4(0.51)$ & $107.7(59.99)$ \\
\hline \multicolumn{8}{|l|}{ Medication group } \\
\hline Total & 86936 & $540.2(10.24)$ & $359.5(7.24)$ & $156.6(0.36)$ & $455.8(2.47)$ & $9.3(0.01)$ & $162.7(1.35)$ \\
\hline \multicolumn{8}{|l|}{ PDD of PPIs } \\
\hline$<$ standard & 76182 & $513.2(10.66)$ & $348.4(7.86)$ & $148.3(0.34)$ & $450.8(2.53)$ & $9.3(0.01)$ & $158.1(1.44)$ \\
\hline standard to $<$ double & 10195 & $702.7(33.59)$ & $426.0(18.49)$ & $213.9(1.45)$ & $495.6(9.04)$ & $9.4(0.03)$ & $191.7(4.03)$ \\
\hline$\geq$ double & 559 & $1251.3(215.52)$ & $658.0(85.56)$ & $235.4(9.49)$ & $412.5(28.29)$ & $9.5(0.12)$ & $254.6(23.30)$ \\
\hline
\end{tabular}

${ }^{a}$ Only subjects who passed 3 months after surgery in each year were analyzed.

PPI, proton pump inhibitor; PDD, prescribed daily dose.

Data are presented as mean (SE).

periods (based on a cutoff of 90 days post-operation), which were considered to reflect the costs of fundoplication and of GERD treatment after surgery, respectively. The total medical costs within
90 days of and over 90 days after surgery were $\$ 2072$ per month (\$6215 for 3 months) and $\$ 108$ per month, respectively. Average medical costs for the medication group were $\$ 163$ per month. $\mathrm{Pa}$ - 
tients prescribed PPIs below the standard dose, between the standard and double doses, and at or above the double dose spent $\$ 158$, $\$ 192$, and $\$ 255$ per month on GERD treatment, respectively.

Additionally, we analyzed the medical costs according to age, sex, and CCI score because the general distributions between the surgery and medication groups were very different. In both the surgery and medication groups, the medical costs were higher in patients with higher CCI scores than in their counterparts with lower scores. However, the medical costs by age groups showed different patterns in the surgery and medication groups. Young adults under age 40 years paid higher costs for GERD treatment compared with middle and older adults in the medication group; whereas, in the surgery group, medical costs were greater in middle and older adults than in young adults (Supplementary Table 1).

Table 4 presents the cost ratios and $95 \%$ CIs for total monthly medical costs for GERD treatment according to therapy type after adjustment for demographics and comorbidities. The medical costs of the surgery group were analyzed in separate GLMs based on 90 days from operation. Total monthly medical costs within 90 days of surgery were 13 times (cost ratio, 12.73; 95\% CI, 9.19-17.64) and 16 times (cost ratio, 16.19; 95\% CI, 12.03-21.78) higher than those of the medication group in the univariate and multivariable models, respectively. When we analyzed the medical costs over 90 days postoperation, there was no statistically significant difference between the medication and surgery groups. We also constructed GLMs using total surgery cases and matching medication samples. Medication samples were selected through 1:4 matching to the surgery cases based on age, sex, and CCI score. Then, we confirmed similar results with the main analysis of Table 4 (Supplementary Tables 2 and 3).

\section{Discussion}

This study provides clear findings of the recent pattern of treatment for GERD patients in Korea. First, a substantial number of GERD patients received PPI medication for at least 12 weeks and this treatment has gradually increased from 2012 to 2016. Over the same period, the number of patients who underwent ARS was significantly lower than that of patients treated with PPIs. Second, based on the PDD calculation for the initial 12 weeks, we found that approximately $10.0 \%$ of long-term PPI users were prescribed more than the standard dose and the proportion also increased with time. Third, medical costs for GERD treatment were 16 times higher in the surgery group than in the medication group within 90 days of ARS; however, medical costs were not significantly different between the surgery and medication groups after 90 days postARS.

The 5-year prevalence of refractory GERD was assumed to

Table 4. Generalized Linear Models for Total Monthly Costs According to Medication or Surgery

\begin{tabular}{|c|c|c|c|c|c|c|c|c|}
\hline \multirow{3}{*}{ Variables } & \multicolumn{4}{|c|}{ Medical expenditure within 90 days $^{\mathrm{a}}$} & \multicolumn{4}{|c|}{ Medical expenditure after 90 days ${ }^{\mathrm{b}}$} \\
\hline & \multicolumn{2}{|c|}{ Univariate model } & \multicolumn{2}{|c|}{ Multivariable model } & \multicolumn{2}{|c|}{ Univariate model } & \multicolumn{2}{|c|}{ Multivariable model } \\
\hline & $\mathrm{CR}(95 \% \mathrm{CI})$ & $P$-value & $\mathrm{CR}(95 \% \mathrm{CI})$ & $P$-value & CR $(95 \% \mathrm{CI})$ & $P$-value & CR $(95 \% \mathrm{CI})$ & $P$-value \\
\hline \multicolumn{9}{|l|}{ Therapy group } \\
\hline Medication & Reference & - & Reference & - & Reference & - & Reference & - \\
\hline Surgery & $12.73(9.19-17.64)$ & $<0.001$ & $16.19(12.03-21.78)$ & $<0.001$ & $0.90(0.58-1.40)$ & 0.648 & $0.84(0.56-1.25)$ & 0.392 \\
\hline \multicolumn{9}{|l|}{ Sex } \\
\hline Men & Reference & - & Reference & - & Reference & - & Reference & - \\
\hline Women & $0.84(0.83-0.85)$ & $<0.001$ & $0.91(0.89-0.92)$ & $<0.001$ & $0.84(0.83-0.85)$ & $<0.001$ & $0.91(0.90-0.92)$ & $<0.001$ \\
\hline \multicolumn{9}{|l|}{ Age (yr) } \\
\hline 20-39 & Reference & - & Reference & - & Reference & - & Reference & - \\
\hline $40-59$ & $0.88(0.84-0.91)$ & $<0.001$ & $0.73(0.70-0.76)$ & $<0.001$ & $0.88(0.84-0.91)$ & $<0.001$ & $0.73(0.70-0.76)$ & $<0.001$ \\
\hline $60-79$ & $0.94(0.90-0.97)$ & 0.001 & $0.68(0.66-0.71)$ & $<0.001$ & $0.94(0.90-0.97)$ & 0.001 & $0.68(0.66-0.71)$ & $<0.001$ \\
\hline$\geq 80$ & $1.04(0.99-1.08)$ & 0.097 & $0.72(0.69-0.75)$ & $<0.001$ & $1.04(0.99-1.08)$ & 0.097 & $0.72(0.69-0.75)$ & $<0.001$ \\
\hline \multicolumn{9}{|l|}{ CCI score } \\
\hline $0-1$ & Reference & - & Reference & - & Reference & - & Reference & - \\
\hline $2-3$ & $1.48(1.45-1.50)$ & $<0.001$ & $1.51(1.48-1.53)$ & $<0.001$ & $1.48(1.45-1.50)$ & $<0.001$ & $1.51(1.48-1.53)$ & $<0.001$ \\
\hline$\geq 4$ & $3.27(3.22-3.33)$ & $<0.001$ & $3.39(3.33-3.44)$ & $<0.001$ & $3.27(3.22-3.33)$ & $<0.001$ & $3.39(3.33-3.44)$ & $<0.001$ \\
\hline
\end{tabular}

${ }^{a}$ Models included the medical expenditure within 90 days of fundoplication in the surgery group.

${ }^{\mathrm{b}}$ Models included the medical expenditure from 90 days after fundoplication in the surgery group.

CR, cost ratio; CCI, Charlson Comorbidity Index. 
be approximately $1.6 \%$ using nationally representative claims data. Although the definition of refractory GERD is controversial, ${ }^{16}$ it is generally described as non-responsiveness of symptoms to PPIs during a treatment period of at least 12 weeks. ${ }^{17}$ Thus, we estimated the prevalence based on the definition of refractory GERD, which is persistent symptoms with long-term use of PPIs for more than 12 weeks. There have been no comparable Korean data on the prevalence of refractory GERD. Previous studies in Korea showed that the GERD prevalence was $7.3 \%,{ }^{3}$ and the proportion of patients with non-responsive GERD ranged from $9.1 \%$ to $26.9 \% .^{18,19}$ These results indicate that approximately $0.7-2.0 \%$ of the total population may have refractory GERD, which is consistent with our results.

In this study, we also observed that the prevalence of refractory GERD, as well as the average PDD of PPIs, increased over time. Numerous empirical studies have suggested the potential adverse effects of long-term use of PPIs, including hypomagnesemia, Clostridium difficile-associated diarrhea, vitamin B12 deficiency, fracture, and dementia. ${ }^{11,20}$ Surgical treatment may be offered as a treatment option or alternative in patients who are concerned about long-term complications of PPI use. ${ }^{21}$

Refractory GERD is one of the most important indications for ARS. Despite some inconsistencies in ARS indications, 2 traditional guidelines, those of the Society of American Gastrointestinal and Endoscopic Surgeons (SAGES) and American Gastroenterology Association (AGA), have commonly recommended that refractory GERD is an indication for ARS. ${ }^{12,21}$ Frazzoni et $\mathrm{al}^{22}$ reported good results with surgical treatment of refractory GERD diagnosed by subgrouping according to impedance-pH monitoring. PPI-responsive GERD is also an indication for ARS. Although the opinions of the AGA and SAGES differ for PPI-responsive GERD, the results of the Long-Term Usage of Esomeprazole vs Surgery for Treatment of Chronic GERD (LOTUS) trial show that heartburn remission is maintained at 5 years post-surgery. ${ }^{23}$

Nevertheless, ARS has been rarely performed in Korea. We found only 40 cases of ARS among 1572839 patients with GERD in our analysis. However, in the US alone, the number of ARS was estimated to be 20000 annually. ${ }^{15}$ Another US study reported that $0.05 \%$ of patients with GERD underwent ARS. ${ }^{14}$ Very low rates of ARS in Korea might be related to concerns about postoperative complications such as dysphagia and flatulence or the lack of awareness of the therapeutic effect of ARS. However, the surgical outcomes, including relief of GERD symptoms (typical and atypical), in Korea were comparable to those in Western countries. ${ }^{24,25}$ Moreover, even if patients took PPIs again after ARS, the PPI response rate was significantly improved. ${ }^{26}$
Our results reveal that medical costs for PPI therapy were high (average monthly cost $=\$ 163$ ). Furthermore, we found that initial medical costs were much higher in the surgery group than in the medication group, but maintenance costs were not significantly different between the groups. Several previous studies showed that the total costs of ARS, including the inpatient care and surgery fees, were high at the beginning of treatment, but gradually decreased. ${ }^{27-29}$

This study has several limitations. First, our data contained a very small number of surgical cases; thus, the results of cost comparisons between surgery and medication need cautious interpretation. Second, the clinical results of each treatment (eg, symptom change and quality of life) were not investigated, because we used secondary data extracted from a national health insurance database. Thus, refractory GERD was defined based on only PPI treatment duration, which may result in over- or under-estimation of prevalence. Third, we analyzed the cross-sectional data per year; therefore, our results could not demonstrate changes in treatment patterns and medical costs over time for more than one year.

To the best of our knowledge, no previous study has reported the national prevalence and treatment patterns of refractory GERD in Korea. Considering the increasing prevalence of long-term use and the high medical costs for PPIs, medical expenditures for GERD can increase in the future. Thus, in order to reduce medical expenditures and improve treatment effectiveness, further studies including sufficient numbers of surgery cases, clinical assessment of efficacy, and long-term follow-up comparing medical and surgical treatments for GERD should be conducted.

\section{Supplementary Materials}

Note: To access the supplementary tables mentioned in this article, visit the online version of Journal of Neurogastroenterology and Motility at http://www.jnmjournal.org/, and at https://doi. org/10.5056/jnm19050.

Financial support: This research was supported by a grant of the Korea Health Technology R\&D Project through the Korea Health Industry Development Institute (KHIDI), funded by the Ministry of Health and Welfare, Republic of Korea (Grant No. HC17C0050).

\section{Conflicts of interest: None.}

Author contributions: Study conception, design, and supervision: Jin-Won Kwon, Joong-Min Park, Sungsoo Park, and Kyung 
Won Seo; data collection, and data analysis and interpretation: Susan Park, Jin-Won Kwon, and Kyung Won Seo; writing of the first draft: Susan Park, Jin-Won Kwon, and Kyung Won Seo; review of medical records, data collection, and data analysis and interpretation: Susan Park, Jin-Won Kwon, Joong-Min Park, Sungsoo Park, and Kyung Won Seo; and review of statistic data: Susan Park and Jin-Won Kwon. All authors were involved in critical revision of the paper.

\section{References}

1. Eusebi LH, Ratnakumaran R, Yuan Y, Solaymani-Dodaran M, Bazzoli F, Ford AC. Global prevalence of, and risk factors for, gastro-oesophageal reflux symptoms: a meta-analysis. Gut 2018;67:430-440.

2. El-Serag HB, Sweet S, Winchester CC, Dent J. Update on the epidemiology of gastro-oesophageal reflux disease: a systematic review. Gut 2014;63:871-880.

3. Kim KM, Cho YK, Bae SJ, et al. Prevalence of gastroesophageal reflux disease in Korea and associated health-care utilization: a national population-based study. J Gastroenterol Hepatol 2012;27:741-745.

4. Min BH, Huh KC, Jung HK, et al. Prevalence of uninvestigated dyspepsia and gastroesophageal reflux disease in Korea: a population-based study using the Rome III criteria. Dig Dis Sci 2014;59:2721-2729.

5. Gawron AJ, French DD, Pandolfino JE, Howden CW. Economic evaluations of gastroesophageal reflux disease medical management. Pharmacoeconomics 2014;32:745-758.

6. Peery AF, Dellon ES, Lund J, et al. Burden of gastrointestinal disease in the United States: 2012 update. Gastroenterology 2012;143:1179-1187, e3.

7. Shaheen NJ, Hansen RA, Morgan DR, et al. The burden of gastrointestinal and liver diseases, 2006. Am J Gastroenterol 2006;101:2128-2138.

8. Park HJ, Park SH, Shim KN, et al. The prevalence and clinical features of non-responsive gastroesophageal reflux disease to practical proton pump inhibitor dose in Korea: a multicenter study. Korean J Gastroenterol 2016;68:16-22.

9. Sandhu DS, Fass R. Current trends in the management of gastroesophageal reflux disease. Gut Liver 2018;12:7-16.

10. El-Serag H, Becher A, Jones R. Systematic review: persistent reflux symptoms on proton pump inhibitor therapy in primary care and community studies. Aliment Pharmacol Ther 2010;32:720-737.

11. Kinoshita Y, Ishimura N, Ishihara S. Advantages and disadvantages of long-term proton pump inhibitor use. J Neurogastroenterol Motil 2018;24:182-196.

12. Katz PO, Gerson LB, Vela MF. Guidelines for the diagnosis and management of gastroesophageal reflux disease. Am J Gastroenterol 2013;108:308-328.

13. Funk LM, Kanji A, Scott Melvin W, Perry KA. Elective antireflux surgery in the US: an analysis of national trends in utilization and inpatient outcomes from 2005 to 2010. Surg Endosc 2014;28:1712-1719.

14. Khan F, Maradey-Romero C, Ganocy S, Frazier R, Fass R. Utilisation of surgical fundoplication for patients with gastro-oesophageal reflux disease in the USA has declined rapidly between 2009 and 2013. Aliment Pharmacol Ther 2016;43:1124-1131.

15. Wang YR, Dempsey DT, Richter JE. Trends and perioperative outcomes of inpatient antireflux surgery in the United States, 1993-2006. Dis Esophagus 2011;24:215-223.

16. Fass R. Proton-pump inhibitor therapy in patients with gastro-oesophageal reflux disease: putative mechanisms of failure. Drugs 2007;67:15211530.

17. Sifrim D, Zerbib F. Diagnosis and management of patients with reflux symptoms refractory to proton pump inhibitors. Gut 2012;61:13401354.

18. Lee ES, Kim N, Lee SH, et al. Comparison of risk factors and clinical responses to proton pump inhibitors in patients with erosive oesophagitis and non-erosive reflux disease. Aliment Pharmacol Ther 2009;30:154164.

19. Kim SE, Kim N, Oh S, et al. Predictive factors of response to proton pump inhibitors in korean patients with gastroesophageal reflux disease. J Neurogastroenterol Motil 2015;21:69-77.

20. Sheen E, Triadafilopoulos G. Adverse effects of long-term proton pump inhibitor therapy. Dig Dis Sci 2011;56:931-950.

21. Stefanidis D, Hope WW, Kohn GP, Reardon PR, Richardson WS, Fanelli RD. Guidelines for surgical treatment of gastroesophageal reflux disease. Surg Endosc 2010;24:2647-2669.

22. Frazzoni M, Piccoli M, Conigliaro R, Manta R, Frazzoni L, Melotti G. Refractory gastroesophageal reflux disease as diagnosed by impedance$\mathrm{pH}$ monitoring can be cured by laparoscopic fundoplication. Surg Endosc 2013;27:2940-2946.

23. Galmiche JP, Hatlebakk J, Attwood S, et al. Laparoscopic antireflux surgery vs esomeprazole treatment for chronic GERD: the LOTUS randomized clinical trial. JAMA 2011;305:1969-1977.

24. Lee JH, Park JM, Han SU, et al. Antireflux surgery in Korea: a nationwide study from 2011 to 2014. Gut Liver 2016;10:726-730.

25. Park JM, Kim BJ, Kim JG, Chi KC. Factors predicting outcomes of laparoscopic Nissen fundoplication for gastroesophageal reflux disease: experience at a single institution in Korea. Ann Surg Treat Res 2017;92:184190.

26. Rickenbacher N, Kötter T, Kochen MM, Scherer M, Blozik E. Fundoplication versus medical management of gastroesophageal reflux disease: systematic review and meta-analysis. Surg Endosc 2014;28:143-155.

27. Faria R, Bojke L, Epstein D, Corbacho B, Sculpher M. Cost-effectiveness of laparoscopic fundoplication versus continued medical management for the treatment of gastro-oesophageal reflux disease based on long-term follow-up of the REFLUX trial. Br J Surg 2013;100:12051213.

28. Goeree R, Hopkins R, Marshall JK, et al. Cost-utility of laparoscopic Nissen fundoplication versus proton pump inhibitors for chronic and controlled gastroesophageal reflux disease: a 3-year prospective randomized controlled trial and economic evaluation. Value Health 2011;14:263273.

29. Bojke L, Hornby E, Sculpher M. A comparison of the cost effectiveness of pharmacotherapy or surgery (laparoscopic fundoplication) in the treatment of GORD. Pharmacoeconomics 2007;25:829-841. 